\title{
BRACHIAL PLEXUS BLOCK FOR URGENT SURGERY IN HEREDITARY ANGIOEDEMA
}

\author{
Catarina Marques ${ }^{1}$, Filipa Carioca ${ }^{1}$, Lúcia Gonçalves ${ }^{1}$, Mauro Mendonça ${ }^{1}$, Rita Poeira ${ }^{1}$, Margarida Casteleira ${ }^{1}$ \\ 1. Centro Hospitalar Lisboa Central; 2. Centro Hospitalar de Leiria; 3. Hospital Central do Funchal
}

\section{BACKGROUND}

Hereditary angioedema (HAE) is a rare genetic disease caused by a deficiency of C1 esterase inhibitor (C1-INH). Mutations in SERPING1 gene can cause low C1-INH levels (type 1) or a non-functioning C1-INH protein (type 2), ultimately leading to bradykinin accumulation and consequent vasodilatation and vascular oedema.

HAE is characterized by recurrent episodes of angioedema of the skin, gastrointestinal tract, and respiratory tract. Flares can be triggered by trauma, infection, cold, pain and emotional stress, among others. Laryngeal oedema with upper airway obstruction and shock are anaesthesiologist's main concerns.

\section{CASE REPORT}

\section{PREANAESTHETIC ASSESSMENT}

54-year-old male, ASA III, proposed for urgent reconstructive left hand surgery after a knife accident.

- Medical history:

- Familial type $2 \mathrm{HAE}$, with previous flares characterized by widespread rash and respiratory distress

- Rheumatoid arthritis in remission

- Urinary retention as a sequelae of lumbar spine surgery

- Myocardial infarction 19 years ago

- No known allergies

- Medication: danazol 100mg a day, etanercept $50 \mathrm{mg}$ a week, methotrexate $10 \mathrm{mg}$ a week and folic acid $5 \mathrm{mg}$ a day

- No predictors of difficult airway found

- 6 hours solids fasting, 2 hours clear liquids fasting

- $1000 \mathrm{U}$ of plasma-derived C1-INH concentrate administered two hours before surgery and another $1000 \mathrm{U}$ kept ready in the OR

\section{INTRAOPERATIVE PERIOD}

- Standard ASA monitoring and a peripheral venous access (20 G)

- Anxiolysis with midazolam $1 \mathrm{mg}$ and fentanyl $100 \mathrm{mcg}$

- Surgical antibiotic prophylaxis with cefoxitin $2 \mathrm{~g}$

- Active warming with a forced-air warming blanket

- Ultrasound-guided axillary brachial plexus block with ropivacaine $0,75 \%$ and lidocaine $2 \%$ (total volume of $30 \mathrm{~mL}$ )

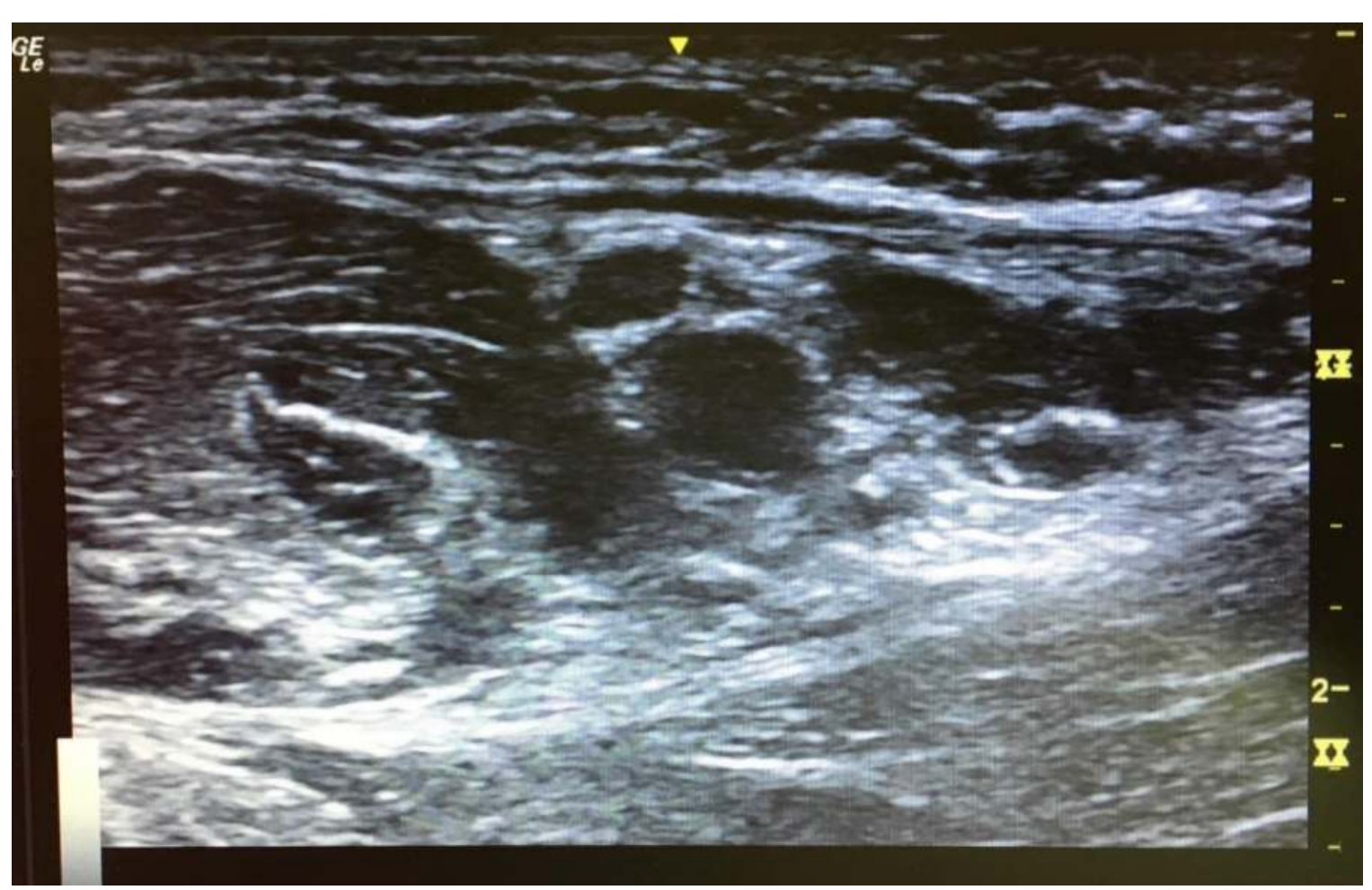

- Emergency drugs were ready and a difficult airway trolley was available throughout the procedure

- Uneventful surgery lasted one hour

\section{POSTANAESTHETIC CARE}

- Vigilance in postanaesthetic care unit

- Multimodal analgesia with paracetamol and metamizole

- No flares or other adverse events occurred

- Discharge home 24 hours after surgery

\section{DISCUSSION}

HAE perioperative management challenges even the most experienced anaesthesiologist. Developing a perioperative plan for prophylaxis, intraoperative management and rescue if a flare occurs is essential, and an immunologist and a pharmacist should be involved.

Potential triggers, like minor trauma and emotional stress, should be avoided during the perioperative period. When HAE patients present with trauma, perioperative flare risk is theoretically higher than in patients proposed for elective surgery.

Preoperatively, even in urgent cases, short-term flare prophylaxis should be performed with C1-INH concentrate one to six hours prior to surgery. At least one dose of C1-INH should be available for rescue.

C1-INH concentrate is the first-line rescue treatment for $\mathrm{HAE}$ flares, as it increases C1-IHN activity. In Portugal there are two C1-INH products derived from human plasma: Berinert $\AA$ and Cinryze $\AA$, and both are approved for treatment and prophylaxis of acute HAE attacks. These products carry the risk of hypersensitivity reactions and venous thromboembolic events.

Androgens, such as danazol, are used for short or long term HAE flare prophylaxis, as they promote hepatic $\mathrm{C} 1-\mathrm{INH}$ synthesis. Dosing strategies and therapeutic duration differ.

Fresh frozen plasma can also be used as a rescue agent and as a prophylactic agent. For prophylaxis, 2-4 units should be administered preoperatively, but care must be taken because infused volumes are high. Also, it can theoretically worsen an attack by replacing depleted complement factors, so it is used as a third-line agent.

Intraoperatively, general anaesthesia and endotracheal intubation should be avoided, although difficult airway equipment should always be available.

Most anaesthetic drugs are safe, but antibiotics, some neuromuscular blockers (succinylcholine and atracurium), meperidine and morphine can cause flares. Adrenaline, corticosteroids and antihistamines are ineffective in angioedema episodes.

Regional anaesthesia should be preferred when suitable. Sedation helps emotional stress management. Multimodal analgesia should be used to achieve optimal pain control, while sparing opioids.

\section{LEARNING POINTS}

$\checkmark$ A multidisciplinary approach optimizes perioperative outcomes in HAE patients. Anaesthetic management in HAE includes identifying and avoiding triggers for angioedema episodes, minimizing airway manipulation and administering prophylactic therapy with $\mathrm{C} 1-\mathrm{INH}$, as surgical trauma is a recognized trigger.

$\checkmark$ A rescue dose of $\mathrm{C} 1-\mathrm{INH}$ should be available to be administered immediately if a flare occurs in the perioperative period. 